\title{
GAMMA-RAY BURST CONSTRAINTS ON THE GALACTIC FREQUENCY OF EXTRA-SOLAR OORT CLOUDS il
}

\author{
J. Michael Shull \\ Joint Institute for Laboratory Astrophysics \\ University of Colorado and National Institute of Standards \& Technology \\ also at CASA, Department of Astrophysical, Planetary, \& Atmospheric Sciences \\ Boulder, CO 80309
}

and

S. Alan Stern

Space Science Department, Southwest Research Institute

6220 Culebra Road

San Antonio, TX 78238

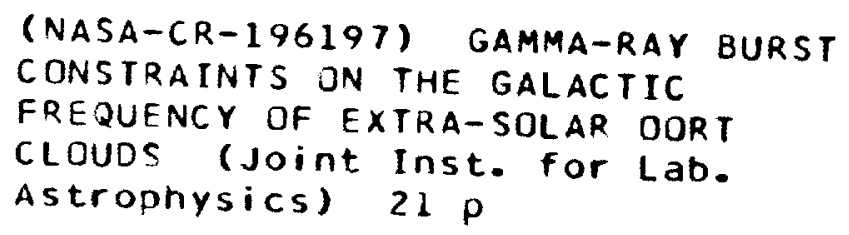

N95-12886

Unclas

$63 / 93 \quad 0027684$

20 Pages

00 Figures

00 Tables

Submitted: March 1994

Revised: June 1994 


\begin{abstract}
With the strong CGRO/BATSE evidence that most $\gamma$-ray bursts do not come from Galactic neutron stars, models involving the accretion of a comet onto a neutron star (NS) no longer appear to be strong contenders for explaining the majority of bursts. If this is the case, then it is worth asking whether the lack of an observed Galactic $\gamma$-ray burst population provides a useful constraint on the number of comets and comet clouds in the Galaxy. Owing to the previously unrecognized structural weakness of cometary nuclei, we find the capture cross sections for comet-NS events to be much higher than previously published estimates, with tidal breakup at distances $R_{b} \approx 4 \times 10^{10} \mathrm{~cm}$ from the NS. As a result, impacts of comets onto field NSs penetrating the Oort Clouds of other stars are found to dominate all other Galactic NS-comet capture rates by a factor of 100 . This in turn predicts that if comet clouds are common, there should be a significant population of repeater sources with (i) a Galactic distribution, (ii) space-correlated repetition, and (ii) a wide range of peak luminosities and luminosity time histories. If all main sequence stars have Oort Clouds like our own, we predict $\sim 4000$ such repeater sources in the Milky Way at any time, each repeating on timescales of months to years. Based on estimates of the sensitivity of the CGRO/BATSE instrument and assuming isotropic $\gamma$-ray beaming from such events, we estimate that a population of $\sim 20-200$ of these Galactic NS-Oort Cloud $\gamma$-ray repeater sources should be detectable by CGRO. In addition, if giant planet formation is common in the Galaxy, we estimate that the accretion of isolated comets injected to the interstellar medium by giant planet formation should produce an additional source of Galactic, non-repeating, events. Comparing these estimates to the $3-4$ soft $\gamma$-ray reeater sources detected by BATSE, one is forced to conclude that (1) comet impacts on NSs are inefficient at producing $\gamma$-rays; or (2) the $\gamma$-rays from such events are highly beamed; or (3) the fraction of stars in the Galaxy with Oort Clouds like our own is not higher than a few percent.
\end{abstract}




\section{INTRODUCTION}

The first widely explored mechanism for producing astrophysical $\gamma$-ray bursts (hereafter denoted GRBs) involved the accretion of comets onto a neutron star (Harwit \& Salpeter 1973: Colgate \& Petschek 1981; Van Buren 1981; Tremaine \& Żytkow 1986; cf. the review by Higdon \& Lingenfelter 1990). Most studies of this mechanism focused on events in which a parent neutron star (NS) accretes bound comets from a comet cloud surrounding its progenitor. However, two main variants on this theme have been reported. In the first (Tremaine \& Żytkow 1986), the parent NS accretes comets from its own Oort Cloud $(\mathrm{OC})$ when the cometary periastra are sufficiently small. This scenario is problematic, however, since it requires that (i) high-mass stars form Oort Clouds, (ii) these stars retain their OCs after the NS-forming event, and (iii) a sufficient number of comets then pass within the NS capture radius to create the rate of observed GRBs. In a second scenario, Pineault (1990) offers a mechanism for removing concerns (ii) and (iii) by having a newly-formed NS capture comets from its own OC as it exits from the center of this cloud, after an asymmetric supernova ( $\mathrm{SN}$ ) explosion.

A small fraction of the GRBs known as Soft Gamma Repeaters (SGRs) exhibit recurrent activity (Atteia et al. 1987), softer spectra (Norris et al. 1991), and a possible association with supernova remnants and pulsars (Kouveliotou et al. 1993; Kulkarni \& Frail 1993). Prompted in part by the evidence for a NS - SGR association, we explore here a third scenario. in which field NSs penetrate the OCs of other stars and accrete comets from these Extra-Solar Oort Clouds (ESOCs). This scenario was independently proposed by Pineault \& Poisson (1989) and Mitrofanov (1990), and later expanded on by Mitrofanov \& Sagdeev (1990) and Pineault (1992).

Our contribution to this scenario is four-fold. (1) In $\S 2$ we re-evaluate the expected NS-ESOC comet accretion rates using more modern cometary and Oort Cloud parameters. Because the capture radius we derive is significantly larger than previously recognized, we find that the event rates produced by this mechanism dominate over captures of comets onto their parent NS by a factor $\sim 10^{2}$. (2) Owing to the larger event rates, in $\S 4$ we find that there should be a significant number of Compton Gamma Ray Observatory (CGRO) detectable Galactic NS-comet accretion events each year, for which we describe the expected characteristics of NS accretion of both ESOC comets and interstellar comets. (3) Later in $\S 4$ we use the paucity of CGRO-detected SGRs to place a significant constraint 


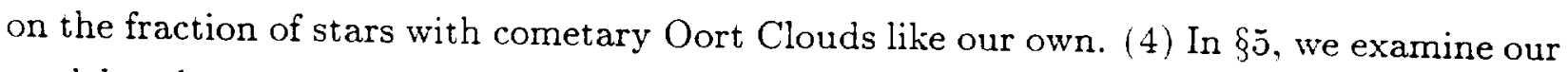
model to determine the number of GRBs that must be detected to constrain the population of interstellar comets. In estimating the capture cross sections and encounter statistics, we also consider the effects of thermal ablation and evaporation in the radiation field of the neutron star (Katz 1986).

\section{ENCOUNTER RATE ESTIMATES}

We begin by computing the encounter rate between field NSs of space density $n_{n}$ s and ESOCs of density $n_{*} f_{o c}$, where $n_{*}$ is the mean local space density of stars and $f_{o c}$ is the fraction of these stars with Oort Clouds. The local Galactic mass density (Bahcall $1984)$ is $(0.185 \pm 0.02) M_{\odot} \mathrm{pc}^{-3}$. of which approximately half can be accounted for by stars. If we adopt a mean stellar mass $\bar{m}=0.5 M_{\odot}$, then $n_{*} \approx 0.2 \mathrm{pc}^{-3}$. For free NSs we adopt a local space density $n_{n s}=2 \times 10^{-4} \mathrm{pc}^{-3}$. corresponding to a total NS population $N_{n s}=2.5 \times 10^{8}$ in the Galactic disk and implying a formation rate of 1 per 40 yrs over $10 \mathrm{Gyr}$. We adopt a typical $\mathrm{NS}$ velocity $v_{n s}=\left(100 \mathrm{~km} \mathrm{~s}^{-1}\right) v_{100}$. Our values for both $n_{n}$ and $v_{n}$, are consistent with recent orbital calculations (Blaes \& Madau 1993) in which pulsars with a bimodal distribution of initial velocities (Narayan \& Ostriker 1990) are injected into a Galactic disk/spheroid of total mass $8.78 \times 10^{10} M_{\odot}$. For an $(\mathbf{r}, \mathbf{v})$ phase-mixed NS vertical distribution with a half-number scale height $\left\langle z_{1 / 2}\right\rangle=280$ pc and a root-mean-square height $z_{r m s}=710 \mathrm{pc}$, a mean stellar mass of $0.5 \mathrm{M}_{\odot}$ implies that the total number of Galactic disk stars is $N_{*}=1.76 \times 10^{11}$, and the effective Galactic volume is $V_{g a l}=9.5 \times 10^{11} \mathrm{pc}^{3}$. The same assumptions give a velocity distribution with $\langle v\rangle=81 \mathrm{~km} \mathrm{~s}^{-1}$ and $v_{r m s}=98 \mathrm{~km} \mathrm{~s}$, which is somewhat slower than the injected pulsar distribution. In $\S 6$ we discuss possible changes in the encounter rates and accretion arising from a recent, upward revision (Lyne \& Lorimer 1994) of the pulsar velocity distribution.

To investigate our scenario quantitatively, we use a simple kinetic model to compute the NS - ESOC encounter rate over the Galactic disk. This yields

$$
\dot{N}_{n s}=n_{n s}\left(\pi R_{o c}^{2}\right) v_{n s} N_{*}=\left(10 \mathrm{yr}^{-1}\right) f_{o c} v_{100} R_{6000}^{2}
$$

Here we have adopted a typical ESOC core radius $R_{o c}=(6000 \mathrm{AU}) R_{6000}$ based on $R_{o c} \approx$ $7500 \mathrm{AU}$ for the dense inner core radius of our own OC (Weissman 1990), scaled to a mean 
stellar mass of $0.5 \mathrm{M} \odot$. Although we could have added the extra parameter of specifying the radial distribution of comets within the ESOC (Pineault \& Poisson 1990), we doubt whether that more sophisticated approach would result in any improvement in the viability of the scaling relations. Importantly, each NS - ESOC penetration will last a sufficiently brief time.

$$
T_{n s} \approx \frac{4 R_{o c}}{3 v_{n s}}=(400 \mathrm{yr}) R_{6000} v_{100}^{-1}
$$

that individual SGR sources should turn off after a few centuries. The intruder NS-ESOC mechanism therefore predicts that one of the 3 - 4 SGRs now known should decrease or cease its burst rate within 50-100 yr.

Combining equations (1) and (2), we find that the total number of intruder NSs in ESOCs spread throughout the disk at any given time should be

$$
N_{n s}=\dot{N}_{n s} T_{n s}=n_{n s} V_{o c} N_{*} f_{o c} \approx 4000 f_{o c} R_{6000}^{3}
$$

independent of the NS velocity, where $V_{\mathrm{oc}}$ is a typical Oort Cloud volume.

By comparison, consider the number of active sources in which a NS accretes comets during post-formation ejection through its own OC (Pineault 1990). For a typical exit velocity of $100 \mathrm{~km} \mathrm{~s}^{-1}$, a NS should spend approximately ( $\left.300 \mathrm{yr}\right) R_{6000}$ departing its own OC core. Because this is $\sim 10^{-7}$ of the $3-5 \mathrm{Gyr}$ age of a typical NS, there should exist an average of $10^{-7} f_{n s, o c} N_{n s}$, or $\sim 25 f_{n s . o c}$ active sources of this type in the disk, where $f_{n s, o c}$ is the fraction of NS progenitors that have Oort Clouds. This ratio can be expressed more simply as

$$
\zeta=10^{7}\left(\frac{V_{o c}}{V_{g a l}}\right)\left(\frac{f_{o c}}{f_{n s, o c}}\right) N_{*} .
$$

This simple comparison demonstrates that there should be $\sim 10^{2}$ times more intruder NSs inside the Oort Clouds of other stars than those in their own Oort Clouds at any given time.

The rate of NS-comet encounters in OCs around parent NSs can only dominate the rate due to NS encounters in the ESOCs of other stars if either: (i) the fraction of NSs with OCs exceeds the average fraction of all stars with OCs by a factor $\sim 10^{2}$, or (ii) NS Oort Clouds have $\sim 10^{2}$ times larger volumes than field-star Oort Clouds. In our view, neither of these alternatives is likely (cf., also Tremaine 1993). Therefore, the intruder 
NS rate of encounters almost certainly the dominant source of NS-comet collisions in the Galaxy. This is our first significant result.

Next, we desire to predict the burst frequency of NS - ESOC sources. As a precursor to that, however, we must compute the NS - comet capture radius, $R_{0}$. We will do so using a new capture mechanism which relies on the tidal breakup of fragile icy comets (see $\S 3$ ). Based on tidal breakup or on the drag introduced by the motion of a conducting body through the NS magnetic field, the capture radii in previous models of NS - comet encounters were of order $3 \times 10^{7} \mathrm{~cm}$ (Van Buren 1981; Tremaine \& Żytkow 1986; Pineault 1990). To investigate the tidal breakup of comets, Colgate \& Petschek (1981) assumed a comet tensile strength $s=10^{10}$ dyn $\mathrm{cm}^{-2}$, characteristic of a Ni/Fe composition, rather than the more fragile "icy mudballs" in the standard comet model (McDonnell et al. 1993). Later, Tremaine \& Żytkow (1986) adopted tensile strengths between $10^{6}$ (Wetherill \& ReVelle 1982) and $10^{\tau}$ dyn $\mathrm{cm}^{-2}$ (Hobbs 1974), which is appropriate for solid ice. However, evidence based on observations of Sun-grazing comets (Sekanina 1982; Hughes 1991) points to strengths in the range of $10^{3}<s<10^{5} \mathrm{dyn} \mathrm{cm}^{-2}$; more recently, the breakup of comet Shoemaker-Levy 9 around Jupiter (Sekanina 1993; Weaver et al. 1994) suggests that $s \approx 10^{3} \mathrm{dyn}^{-2}$ (Scotti \& Melosh 1993). In what follows, we conservatively adopt $s=\left(10^{5}\right.$ dyn $\left.\mathrm{cm}^{-2}\right) s_{5}$.

Comets in our Solar System have typical masses between $10^{16} \mathrm{~g}$ and $10^{18} \mathrm{~g}$, and consist of comparable amounts of water ice and heavy elements (Weissman 1990). We consider a canonical comet of mass $m_{c}=\left(10^{17} \mathrm{~g}\right) m_{17}$, density $\rho_{c}=\left(1.0 \mathrm{~g} \mathrm{~cm}^{-3}\right) \rho_{0}$, and radius $R_{c}=(2.88 \mathrm{~km}) m_{17}^{1 / 3} \rho_{0}^{-1 / 3}$ approaching a NS of mass $M_{n s}=1.4 M_{\odot}$. The tidal breakup radius for such comets in the NS gravitational field is

$$
R_{b}=\left(\rho_{0} R_{c}^{2} G M_{n s} / s\right)^{1 / 3} \approx\left(5.4 \times 10^{10} \mathrm{~cm}\right) m_{17}^{2 / 9} s_{5}^{-1 / 3} \rho_{0}^{1 / 9} .
$$

If we consider the gravitational focussing of the comet's orbit by the NS, a typical approach velocity $v_{n}, R_{b}$ corresponds to an impact capture parameter of

$$
R_{0}=\left(2 G M M_{n} R_{b}\right)^{1 / 2} / v_{n s} \approx\left(4.5 \times 10^{11} \mathrm{~cm}\right) m_{17}^{1 / 9} s_{5}^{-1 / 6} \rho_{0}^{1 / 18} v_{100}^{-1} .
$$

Most evidence suggests that comets are a conglomerate of fragments with varying tensile strengths, including gravel, dust, and ice particles. The weakest fragments will be broken at the largest distances, followed by a cascade down to the size of particles of sufficiently 
small size and high tensile strength (i.e., $10^{7-9} \mathrm{dyn}_{\mathrm{cm}}^{-2}$ ) to resist the tidal forces. We expect this cascade to occur over time scales comparable to the sound-wave crossing time of the solid fragments ( seconds). Because the strength of the individual fragments is expected to be much weaker than ice, the tidal cascade should not cease until the fragments reach the size of strong (i.e.. molecular) grains of ice and dust. We show in $\S 3$ that these grains will quickly evaporate and become ionized in the NS radiation field to create a plasma.

Once the cascade to smaller fragments begins, the debris area rises substantially, so that significant radiation can be absorbed. The difficulty with this cascade from km-sized comets to cm-sized fragments is that $R_{b} \propto\left(R_{c}^{2} / s\right)^{1 / 3}$. Thus, smaller fragments eventually become stable against tidal forces, but the factor of 100 range in tensile strength, $s=10^{3-5}$ dyn $\mathrm{cm}^{-2}$ only translates into a factor of 5 in breakup size. However, because the process of fragmentation is incompletely understood and may involve fragment collisions or forces of evaporative jetting, some unknown fraction of the fragments will probably continue on hyperbolic trajectories.

Again adopting a kinetic model, we predict that the comet - NS collision rate in each of the $4000 f_{o c}$ active Galactic NS - ESOC sources is

$$
\dot{N}_{c}=n_{c}\left(\pi R_{0}^{2}\right) v_{n s}=\left(0.65 \mathrm{yr}^{-1}\right) N_{13} R_{6000}^{-3} v_{100}^{-1} m_{17}^{2 / 9} s_{5}^{-1 / 3} \rho_{0}^{1 / 9}
$$

where $n_{c}$ is the average number density of comets in the comet cloud, $R_{0}$ is the NScomet capture radius, and $N_{13}$ is the number of comets per OC in units of $10^{13}$, the estimated population for our own OC (Weissman 1990; Mumma, Weissman, \& Stern 1993). Multiplying equations (3) and (7), we see that the total event rate in the Galaxy is of order $2600 \mathrm{yr}^{-1}$, some fraction of which are near enough to be detectable (see $\S 4$ ).

Clearly, with a typical encounter timescale of a comet every $\sim 1-2$ yrs, field NS penetrations of ESOCs will produce repetitive, spatially correlated accretion events. Because the details of cometary breakup and accretion onto a NS are strongly dependent on the structural properties of the nucleus, the NS mass, and the encounter geometry (e.g., whether or not some debris is trapped in a temporary orbit in the NS magnetosphere), we expect a wide range of accretion luminosity time histories to result, ranging from milliseconds to minutes. 


\section{COMET - NEUTRON STAR COLLISION CROSS SECTIONS}

As noted in $\S 2$, previous NS-comet tidal disruption studies adopted tensile strengths that are $3-5$ orders of magnitude larger than those in the standard "icy mudball" comets (Mumma et al. 1093; Sekanina 1993) seen around our Sun. The result of a NS-comet encounter is a debris cloud of mass $m_{c}$, initially moving along the original hyperbolic approach trajectory at breakup, of velocity

$$
v_{b}=\frac{R_{0} v_{n s}}{R_{b}} \approx\left(830 \mathrm{~km} \mathrm{~s}^{-1}\right) m_{17}^{-1 / 9} s_{5}^{1 / 6} \rho_{0}^{-1 / 18} .
$$

For impact parameters less than $R_{0}$, the comet will penetrate inside $R_{b}$, where it will break into a number of smaller pieces, each with a random velocity about $v_{b}$ provided by differential tidal acceleration and heating-related jetting forces. Charged particles will be affected by the NS magnetosphere. In this section we calculate the tidal capture radius for icy mudball comets. We then discuss the fate of this tidal debris in the strong gravitational and magnetic fields, and the intense X-ray/UV radiation environments of NSs.

\subsection{Ablation and Evaporation}

We now show that during the inbound portion of the encounter, of duration

$$
t_{\text {enc }}=\left(\pi R_{b} / v_{b}\right)=(2000 \mathrm{~s}) m_{17}^{1 / 3} s_{5}^{-1 / 2} \rho_{0}^{1 / 6},
$$

significant evaporation of the tidally-produced fragments occurs in the NS radiation field. Once the breakup process begins, the surface area exposed to NS heating increases. The flux of NS radiation is set by its surface area and surface temperature; the latter is determined by accretion from the interstellar medium to be least $3 \times 10^{5} \mathrm{~K}$ and perhaps as high as $10^{6} \mathrm{~K}$ (Wang \& Sutherland 1994). If we parameterize $T_{\mathrm{ns}}=\left(10^{5.5} \mathrm{~K}\right) T_{5.5}$ and $L_{\mathrm{ns}}=\left(7 \times 10^{30} \mathrm{ergs} \mathrm{s}^{-1}\right) T_{5.5}^{4}$, the blackbody radiative flux at a distance $R=R_{b}$ from a $10 \mathrm{~km}$ radius NS is

$$
F_{\mathrm{ns}}=\frac{L_{\mathrm{ns}}}{4 \pi R_{b}^{2}}=\left(2 \times 10^{8} \mathrm{ergs} \mathrm{cm}^{-2} \mathrm{~s}^{-1}\right) T_{5.5}^{4} m_{17}^{-4 / 9} s_{5}^{2 / 3} \rho_{0}^{-2 / 9} .
$$

For large fragments, in which thermal conduction across their diameter can be neglected, the equilibrium temperature is

$$
T_{\mathrm{c}}=\left(\frac{R_{\mathrm{ns}}}{R_{b}}\right)^{1 / 2}\left(\frac{1-A}{4 \epsilon}\right)^{1 / 4} T_{\mathrm{ns}} \approx(980 \mathrm{~K}) T_{5.5} m_{17}^{-1 / 9} s_{5}^{1 / 6} \rho_{0}^{-1 / 18}
$$


for a typical comet albedo $A \approx 0.04$ and emissivity $\epsilon \approx 0.9$ at $R=R_{b}$. At this high temperature, significant sublimation of cometary ice will occur, thereby limiting the surface temperature. By equating the latent heat flux of sublimation to the radiative heating flux, for values given in equation (10) with scaling factors set to unity, we estimate that the actual temperature is $\sim 240 \mathrm{~K}$, with a mass loss rate per unit area $\sim 0.002 \mathrm{~g} \mathrm{~cm}^{-2} \mathrm{~s}^{-1}$. This is sufficient, over the encounter time, to erode several centimeters from the surfaces of all comet fragments.

To estimate the fragment size that can sublimate completely, we first calculate the "fluence" (energy $\Delta E$ per projected area $\mathrm{A}$ ) delivered to a single comet over the total encounter. For an orbit with specific angular momentum $R_{0} v_{n s}$, this is given by

$$
\frac{\Delta E}{A}=\frac{L_{\mathrm{ns}}}{2 R_{0} v_{\mathrm{ns}}}=\left(8 \times 10^{11} \mathrm{ergs} \mathrm{cm}{ }^{-2}\right) T_{5.5}^{4} m_{17}^{-1 / 9} s_{5}^{1 / 6} \rho_{0}^{-1 / 18}
$$

which is sufficient (Katz 1986) to ablate cometary surfaces to a depth

$$
\Delta R=\frac{(\Delta E) / A}{4 \rho_{\mathrm{c}} h(T)}=(7 \mathrm{~cm}) T_{5.5}^{4} m_{17}^{-1 / 9}{ }_{s_{5}}^{1 / 6} \rho_{0}^{-1 / 18},
$$

where $h(T) \approx 3 \times 10^{10} \mathrm{ergs}^{-1}$ is the steam enthalpy at $T \approx 240 \mathrm{~K}$. Radiative cooling can be shown to be negligible under these conditions.

The ablation depth is insensitive to the comet parameters, but is extremely sensitive to the NS radiation temperature, which is set by the rate of interstellar accretion, as discussed further in $\S 3.2$. The NS surface temperature of $10^{5.5} \mathrm{~K}$ corresponds to a mass accretion rate $\dot{M}=8 \times 10^{10} \mathrm{~g} \mathrm{~s}^{-1}$ onto the entire NS surface (10 km radius) at $10 \%$ efficiency. Such accretion rates are entirely consistent with the detailed modeling of NS accretion from the ISM (Blaes \& Madau 1993), although large differences in the accretion rate and X-ray luminosity can arise for different phases of the ISM and from polar-cap accretion. The population of SGRs might, for example, arise from NSs accreting from regions with higher than average density. Old NSs might also be expected to have somewhat lower magnetic fields, suggesting that the higher $\mathrm{X}$-ray luminosities and radiation temperatures associated with polar-cap accretion are not present.

In the NS radiation field assumed here, fragments of radii $\sim 0.1 \mathrm{~m}$ will be completely vaporized. Fragments as large as several meters could evaporate only around NSs at $10^{6} \mathrm{~K}$. The fate of the larger chunks of the comet cascade is uncertain and probably depends on processes that occur during the tidal breakup, such as ablation, jetting, and 
fragment collisions. The limiting fragment size may drop from $1 \mathrm{~km}$ to $1 \mathrm{~m}$ in proportion to $\left(R^{2} / s\right)^{1 / 3}$. These processes may be able to bridge the gap between the initial $\mathrm{km}$-sized fragments of the comet and the sub-meter sized fragments than can evaporate in the NS radiation field. Thus, a significant fraction of the comet mass may be able to attach to the NS magnetic field lines and accrete to the NS. From equations (12) and (13), we note that significant fragment ablation (to depths $\Delta R$ on the order of meters) requires fluences $\sim 10^{13}$ ergs $\mathrm{cm}^{-2}$ corresponding to a NS surface temperature $10^{5.8} \mathrm{~K}$ at an impact parameter $R_{0}$ given by equation (6). Alternatively, we can re-write the impact parameter necessary for a given $\Delta R$ as

$$
R_{0}=\left(3 \times 10^{10} \mathrm{~cm}\right) T_{5.5}^{4} v_{100}^{-1} \rho_{0}^{-1}\left(\frac{1 \mathrm{~m}}{\Delta R}\right) .
$$

Thus, including gravitational focussing, we have demonstrated that comets of tensile strength $s=10^{5}$ dyn $\mathrm{cm}^{-2}$ are tidally disrupted for impact parameters $R_{0} \leq 4.5 \times 10^{11}$ $\mathrm{cm}$. For impact parameters $R_{0}$ ranging from $4 \times 10^{11} \mathrm{~cm}$ inward to $3 \times 10^{10} \mathrm{~cm}$, increasing amounts of the tidal fragments will be ablated. In the intense radiation field near the NS, the molecules produced by the sublimation of the cometary ice and dust fragments will be quickly dissociated and ionized. The photoionization time of $\mathrm{H}_{2} \mathrm{O}$ and its primary daughter products $\mathrm{OH}$ and $\mathrm{H}$ at any distance $\mathrm{R}$ from the NS is quite short,

$$
t_{i} \approx(3 \mathrm{~s})\left(\frac{R}{R_{b, 0}}\right)^{2}
$$

where $\mathrm{R}_{b, 0}=4.5 \times 10^{10} \mathrm{~cm}$, as given in Equation (6). The resulting plasma should consist of ions that can attach to the NS magnetic field lines and accrete to the NS surface, since the effects of NS radiation pressure can be shown to be small.

\subsection{Magnetospheric Capture}

The processes governing the magnetospheric penetration of this debris cloud and its subsequent accretion onto the NS now deserves discussion. To gain some insight into which processes dominate, we estimate criteria for: (1) the debris cloud avoiding magnetospheric deflection; and (2) the plasma attaching to the magnetic field lines. We assume that the plasma from the comet is quickly heated to temperatures $\sim 10^{4} \mathrm{~K}$ by the UV/X-ray radiation from the NS. For a silicon/oxygen plasma, this temperature corresponds to a mean thermal expansion speed of $v_{\exp } \approx\left(3 \mathrm{~km} \mathrm{~s}^{-1}\right) v_{3}$; for $\mathrm{H}$ the thermal expansion speed 
is $\sim 10 \mathrm{~km} \mathrm{~s}^{-1}$. Over the characteristic duration of the encounter. $t_{\mathrm{enc}}$, this plasma cloud will expand to fill a sphere of radius $R_{\exp } \approx v_{\exp } t_{\text {enc }}$.

To set the first criterion for whether this diluted debris cloud is deflected from the magnetosphere or instead penetrates to the NS surface, we compare the ratio of the ram pressure of the plasma debris cloud, $\rho V^{2}$, to the magnetic energy density, $\left(B^{2} / 8 \pi\right)$, at the breakup radius $R_{b}$. For the NS magnetosphere, we assume a dipole magnetic field, $B(R)=B_{0}\left(R_{\mathrm{ns}} / R\right)^{3}$, typical of an old NS. Current studies show that these fields may be high. Whereas the majority of early pulsar studies assumed that the initial large $\left(10^{12.5} \mathrm{G}\right)$ magnetic field decays exponentially on a time scale of order $10^{7 \pm 0.2}$ yr (Gunn \& Ostriker 1970; Lyne, Manchester, \& Taylor 1985), more recent investigations (Narayan \& Ostriker 1990) show that the form of the field decay (exponential or power-law) is uncertain. There also have been suggestions for both a long-lived residual component $B \leq 10^{10} \mathrm{G}$ (Kulkarni 1086 ) and for little or no field decay in isolated NSs over $10^{8} \mathrm{yr}$ (Bhattacharya et al. 1992: Chanmugam 1992). We adopt a surface field parametrization $B_{0}=\left(10^{10} \mathrm{G}\right) B_{10}$, realizing that the factor $B_{10}$ may range between $10^{-2}$ and $10^{2}$.

For the ram pressure of the comet debris cloud, we assume that the plasma debris cloud moves at bulk velocity $v=v_{b}$ and that the debris cloud density is $\rho_{b}=$ $m_{c} /\left[(4 \pi / 3)\left(v_{\exp } t_{\text {enc }}\right)^{3}\right]$. The much lower random velocity of the solid fragments is produced by fluctuations in the tidal acceleration. Since most of the fragments are vaporized, we can use the fluid approximation to evaluate the ratio of ram pressure to magnetic pressure at $R_{b}$,

$$
\frac{\rho_{b} v_{b}^{2}}{B^{2} / 8 \pi}=\left(5 \times 10^{15}\right) m_{17}^{10 / 9} s_{5}^{-1 / 6} \rho_{0}^{1 / 18} B_{10}^{-2} v_{3}^{-3} .
$$

Because the above ratio is much greater than unity for any likely NS magnetic field, comet mass, density, and tensile strength, the plasma debris should easily penetrate the NS magnetosphere. Of course, some of the larger comet fragments may escape full vaporization, and therefore avoid capture by the NS magnetosphere.

We now investigate the second criterion, which governs whether the accreted plasma attaches to the magnetic field lines of the NS. The Larmor radius of a debris fragment of mass $N m_{p}$, charge $Z e$, and gyrovelocity $v_{b}$ arising from the original comet of mass $m_{c}$ is given by

$$
R_{L}=\frac{\left(N m_{p} c v_{b} R_{b}^{3}\right)}{\left(Z e B_{0} R_{\mathrm{ns}}^{3}\right)} \approx\left(1 \times 10^{8} \mathrm{~cm}\right)\left(\frac{N}{Z}\right) m_{17}^{5 / 9} s_{5}^{-5 / 6} \rho_{0}^{5 / 18} B_{10}^{-1} .
$$

This gyro-radius is considerably less than $R_{b}$ for fields $B_{0} \geq 10^{10} \mathrm{G}$. and can be reduced 
for values of $N / Z$ appropriate to a vaporized fragment. Therefore, the accreting plasma will follow the magnetic field lines down to the NS surface, once it penetrates the magnetosphere.

We evaluate the radius. $R_{M}$, of the magnetosphere first for a NS accreting at rest from a static ambient ISM with isothermal sound speed $c_{s}=\left(10 \mathrm{~km} \mathrm{~s}^{-1}\right) c_{10}$, and then for a NS moving at velocity $v_{n s} \gg c_{s}$. For an isolated. low-velocity NS, we assume isothermal, spherical accretion flow and equate the ram pressure, $\rho v^{2}=\left(\rho_{s} c_{s}^{2}\right)\left(r / R_{s}\right)^{-5 / 2}$, of the accreting matter inside the sonic radius, $R_{s}=\left(G M_{n s} / 2 c_{s}^{2}\right)$, to the magnetic pressure, $\left(B_{0}^{2} / 8 \pi\right)\left(r / R_{n s}\right)^{-6}$. The density at the sonic radius, $\rho_{s}=e^{3 / 2} \rho_{\infty}$, can be related to the mass infall rate, $\dot{M}=\left(e^{3 / 2} / 4\right)(4 \pi) \rho_{\infty}\left(G M_{n s}\right)^{2} / c_{s}^{3}$, and to the NS luminosity, $L_{n s}=$ $\dot{M}\left(\eta_{a} c^{2}\right)$, with $\eta_{a} \approx 0.1$. We then find a magnetospheric radius independent of $c_{\boldsymbol{s}}$,

$$
R_{M}=\left[\frac{B_{0}^{2} R_{n s}^{6} \eta_{a} c^{2}}{L_{n s}\left(2 G M_{n s}\right)^{1 / 2}}\right]^{2 / 7}=\left(1.2 \times 10^{9} \mathrm{~cm}\right) B_{10}^{4 / 7} T_{5.5}^{-8 / 7}
$$

where we have expressed $L_{n s}$ in terms of the NS effective temperature. For the more likely case where $v_{n s} \gg c_{s}$, one substitutes $v_{n s}$ for $c_{s}$ (Hoyle \& Lyttleton 1941). The formulae for accretion radius and $R_{M}$ may be written in terms of the NS luminosity, similar to eq. (18), or in terms of ambient density and NS velocity,

$$
R_{\mathrm{acc}} \approx \frac{G M_{n s}}{v_{n s}^{2}}=\left(1.9 \times 10^{12} \mathrm{~cm}\right) v_{100}^{-2}
$$

and

$$
R_{M}=\left[\frac{B_{0}^{2} R_{n s}^{6} v_{n s}^{3}}{8 \pi \rho_{0}\left(G M_{n s}\right)^{5 / 2}}\right]^{2 / 7}=\left(4 \times 10^{9} \mathrm{~cm}\right) B_{10}^{4 / 7} v_{100}^{6 / 7} n_{0}^{-2 / 7} .
$$

Evidently the tidal breakup and fragment vaporization occur at distances $\sim 10^{10-11} \mathrm{~cm}$ from the NS, which is larger than the magnetospheric radii for old NSs accreting at a standard rate from the ISM. Because the Larmor radii of the evaporated fragments are much less than both $R_{b}$ and $R_{M}$, it is likely that the ablated and ionized debris attaches to the field lines of the NS. Although the comet debris cascade will be complex, it is possible that significant amounts of the cometary dust and ice are broken up, sublimated and ionized so that they penetrate the NS magnetosphere and accrete onto the NS to produce a $\gamma$-ray burst as previously explored (e.g., Colgate \& Petschek 1981). If the capture radius is $R_{0} \approx 4 \times 10^{11} \mathrm{~cm}$, as argued in equation (6), then the encounter frequency is 
interestingly large $\left(\sim 1 \mathrm{yr}^{-1}\right)$. If the effective $R_{0}$ is a factor of $4-5$ smaller, owing to incomplete evaporation of the comet fragments, the encounter rate drops to a value

$$
\dot{N}_{c}=\left(0.033 \mathrm{yr}^{-1}\right) N_{13} R_{6000}^{3}\left(\frac{R_{0}}{10^{11} \mathrm{~cm}}\right)^{2}
$$

In that case, the repetition frequency of bursts might be dominated by bound comet debris returning to the NS on later orbital passages.

\section{COMET - NS IMPACT DETECTABILITY AND CHARACTERISTICS}

We now consider the detectability of cometary accretion events onto NSs. For a comet of mass $m_{c}=\left(10^{17} \mathrm{~g}\right) m_{17}$, the energy released is a significant fraction of the comet's rest mass, and may be written

$$
E=\eta m_{c} c^{2} \approx\left(9 \times 10^{36} \text { ergs }\right) m_{17}\left(\frac{\eta}{0.1}\right)
$$

Here, $\eta$ is the net $\gamma$-ray conversion efficiency of the collision, normalized to the comet rest-mass energy. Since the gravitational potential energy at the NS surface is about $20 \%$ of the rest-mass energy, $\eta=0.1$ corresponds to conversion of half the kinetic energy of infall into $\gamma$-rays. Values a factor of 5 lower have also been used (Tremaine \& Żytkow 1986). Since the CGRO/BATSE $\gamma$-ray burst detector has sensitivity to burst fluxes down to $F_{\text {min }}=3 \times 10^{-8}$ ergs cm $\mathrm{cm}^{-2} \mathrm{~s}^{-1}$ (Schaefer 1993a; Meegan et al. 1992), a comet accretion event onto a NS could be detected to a distance of

$$
D_{\max }=\left(\frac{E}{4 \pi F_{\min } \delta T}\right)^{1 / 2} \approx(1.6 \mathrm{kpc}) m_{17}^{1 / 2}\left(\frac{\eta}{0.1}\right)^{1 / 2}(\delta T)^{-1 / 2}
$$

where $\delta T$ is the burst duration timescale in seconds. For $\delta T=0.1 \mathrm{~s}$ (Kouveliotou et al. 1993; Higdon \& Lingenfelter 1990), we obtain $D_{\max }=5 \mathrm{kpc}$. Although our poor knowledge of $\eta$ makes these estimates highly uncertain, both the $1.6 \mathrm{kpc}$ and $5 \mathrm{kpc}$ detection distance scales are small compared to the radius of the Galactic disk, but large enough to reach neighboring spiral arms. Since $D_{\max }$ is comparable to the vertical scale height of the "thick stellar disk" and the 710 pc thickness of the phase-mixed NS population (Blaes \& Madau 1993), NS-comet gamma ray bursts produced by field NS intruding into ESOCs should 
exhibit a Galactic distribution. Since this is contradiction to the BATSE observations for the general GRB population. we can obtain a constraint on $f_{o c}$ for the SGR population.

Assuming that the spatial distribution of NSs and OCs are each homogeneous over the Galactic disk, then our estimate of $\sim 4000 f_{o c}$ active NS - OC sources in a disk of radius $12 \mathrm{kpc}$, detectable to $1.6 \mathrm{kpc}$, implies 70 foc BATSE-detectable, repeating NS $\mathrm{OC}$ sources at any given time. For the more relevant $\delta T=0.1 \mathrm{~s}$ SGR burst timescales detected by BATSE, corresponding to a detection limit of $5 \mathrm{kpc}$, we predict $\sim 700 f_{o c}$ BATSE-detectable, repeating NS - OC sources at any given time. This is our second result.

Only three (Hurley 1993) or four (Wang \& Lingenfelter 1993) SGRs have been identified to date. Two of these sources can be associated with NSs: SGR 1806-20 is associated with a Galactic pulsar and supernova remnant (Kulkarni \& Frail 1993), while SGR 0525-66 has been associated with the LMC supernova remnant N49 (Mazets et al. 1982; Helfand \& Long 1979) and a compact X-ray source, possibly a pulsar (Rothschild et al. 1994). Recurrent activity over more than $20 \mathrm{yrs}$ in the repeater SGR $1900+14$ (Kouvelioutou et al. 1993) and repetitive soft $\gamma$-ray bursts from GB 790107 (Livio \& Taam 1987) lend further support to suggestions that SGRs are related to NSs. Thus, unless no $\gamma$-rays are produced in comet accretion mechanism or unless the $\gamma$-rays are sufficiently beamed that few events are detectable from Earth, the occurrence fraction of stars with Oort Clouds similar to our own is not high. As a result, we estimate that for $D_{\max }=1.6 \mathrm{kpc}$, no more than $3 / 70 \approx 4 \%$ of all stars have comet clouds similar in population and structure to our Oort Cloud. This is our third result. Because this limit depends crucially on $D_{\max }$, this figure is only illustrative.

If, in the future, the already-detected SGRs are found to reside beyond the $\sim 1.6$ kpc upper limit on the (BATSE) NS-comet detection distance, then $f_{o c}$ can be even more tightly constrained to $<1 \%$. Since the formation of OCs like our own clearly depends on the presence and astrocentric distribution of giant planets in the distant solar system (cf., Weissman 1990; Tremaine 1993), our results suggest that such solar system architectures could be rare. In the same regard, it is worth noting that even if the impacting comets were as massive as $10^{18} \mathrm{~g}$, their collision with a NS would not likely be detected by BATSE in the LMC (e.g., the SGR tentatively identified with the N49 supernova remnant). On the other hand, detections of massive-comet captures by NSs in the nearest globular clusters, at $3-10 \mathrm{kpc}$, would be possible. 


\section{NS COLLISION RATES WITH INTERSTELLAR COMETS}

In addition to collisions between ESOCs and NSs. field NSs can also accrete free comets residing in the interstellar medium (ISM). The best estimates for our OC (Weissman 1990: Tremaine 1993) predict that between 2 and 5 times the number of comets injected into bound orbits were ejected hyperbolically, with Jupiter and Saturn being the primary scattering centers for interstellar comets. It is therefore widely expected that the ISM contains a significant population of comets (Sekanina 1976; McGlynn \& Chapman 1989; Stern 1990).

If there are $\alpha>1$ interstellar comets (ISCs) for every OC comet in the Galaxy, then field NSs should encounter interstellar comets at $\alpha$ times the rate of $\mathrm{OC}$ comets. Adopting the same formalism as in Eqns. [3] and [7] above, we estimate that the ISC source should produce $2600 \alpha f_{o c}$ NS-comet collisions $\mathrm{yr}^{-1}$ in the Galaxy. Thus, if comet clouds like our own are rare, there should exist $\alpha=2-5$ times as many isolated (i.e., non-repeating) Galactic GRB sources due to NS-interstellar comet collisions as there are $\mathrm{NS}-\mathrm{OC}$ repeaters. However, for the same capture radius as in equation (6), we predict that for each NS, the mean time between interstellar comet collisions is in excess of $10^{4} \mathrm{yr}$. Because dynamical mixing will cause the interstellar comets to be smoothly distributed in the ISM, the space density of interstellar comets will be $\sim\left(2 \times 10^{-5}\right) \alpha$ times less than that of OC comets, and, unlike NS - ESOC collisions, collisions between NSs and ISCs will not be spatially clustered. This is an important distinction.

We estimate that a Galactic population of between $90 f_{o c} \mathrm{yr}^{-1}$ and $230 f_{o c} \mathrm{yr}^{-1}$ nonrepeating sources produced by NS collisions with ISCs should be detectable in the BATSE dataset for $\alpha=2-5$ and a BATSE burst detection limit of $1.6 \mathrm{kpc}$. These sources should exceed the SGR bursts by a factor $\alpha=2-5$. As demonstrated by Smith \& Lamb (1993) and Syer \& Saha (1994), one can hide up to $20 \%$ of the population of BATSE-detected GRBs distributed in the Galactic disk and remain consistent with current BATSE isotropy measurements. This would permit up to 80 possible Galactic NS - ISC events $\mathrm{yr}^{-1}$ to reside in the BATSE dataset, currently (June 1994) totaling over 1000. Therefore, as our fourth result, we conclude that the present BATSE dataset does not allow the population of ISCs to be meaningfully constrained. However, over the next several years, as the number of GRBs in the BATSE catalogs increases, we could expect factor of three improvements on the allowable population of Galactic GRBs. This should permit the number of ISCs to be 
constrained.

\section{NEUTRON STAR VELOCITY DISTRIBUTION}

In the calculations above. we scaled the encounter rates to a fiducial NS space velocity $v_{n s}=\left(100 \mathrm{~km} \mathrm{~s}^{-1}\right) v_{100}$, based on orbital calculations (Blaes \& Madau 1993) of pulsars injected into the Galactic potential with a bimodal distribution of initial velocities ranging from 50 to $400 \mathrm{~km}^{-1}$ (Lyne, Anderson, \& Salter 1982; Narayan \& Ostriker 1990). However, new results indicate that pulsar initial velocities may be much higher. A recent re-evaluation (Lyne \& Lorimer 1394) of the radio pulsar velocity distribution, taking into account a new distance scale, new proper motions, and selection effects, concludes that the mean pulsar space velocity at birth may be as high as $450 \pm 90 \mathrm{~km} \mathrm{~s}^{-1}$. There have also been suggestions (Kulkarni et al. 1994; Rothschild, Kulkarni, \& Lingenfelter 1994) that two SGR burst sources are associated with pulsars and neutron stars with extremely high space velocities, $v_{n}>500 \mathrm{~km} \mathrm{~s}^{-1}$. Therefore, it is worth considering the differences in our scenario for high-velocity NSs.

Looking back to $\S 2$ (eqs. [1] - [3]), we note that the NS - ESOC encounter rates will increase linearly with $v_{100}$, but the encounter durations will drop as $v_{100}^{-1}$. Therefore, the expected total number of NSs intruding in ESOCs will remain invariant at around $4000 f_{o c}$. However, the rate of comet - NS encounters in each of these sources (see eq. [7]) will decrease as $v_{100}^{-1}$. If the SGR population turns out to be associated with NSs of abnormally large space velocities $\left(v_{n s} \geq 500 \mathrm{~km} \mathrm{~s}^{-1}\right)$ their accretion rates from the ISM and their surface temperatures will drop. For accretion with $v_{n s} \gg c_{s}$, the mass accretion rate (see eq. [19]) and accretion luminosity scale as $v_{100}^{-3}$, and the surface temperature therefore will drop as $v_{100}^{-3 / 4}$. The expected decrease in NS luminosity would result in considerably less radiative evaporation of comets during the shorter encounters (see eq. [13]).

Thus, we conclude that, although the total number of "intruder NS" sources will remain the same throughout the Galaxy, the individual encounters between very fast, NSs and ESOCs will several times be less frequent, and the radiative ablation and subsequent accretion of cometary fragments will be much lower. It is therefore difficult to see how these encounters would be likely to make $\gamma$-ray bursts. As noted above, the more likely 
sources, for our model of GRBs, are the older. slower population of NSs that have felt the gravitational potential of the Galaxy.

\section{SUMMARY}

The fact that the observed distribution of BATSE sources is both isotropic on the sky and volume limited (Meegan et al. 1992) has been used to suggest that most GRBs are not derived from Galactic sources (Paczynski 1991. 1993). Unless recent hints of small anisotropies in the distribution of intermediate count-rate GRBs (Quashnock \& Lamb 1993: Rutledge \& Lewin 1993) turn out to be statistically significant (Schaefer 1993b), the apparent GRB isotropy argues that the majority of the bursters do not result from collisions between Galactic comets and NSs.

We have shown that the rate of NS - comet collisions should be dominated by field NSs colliding with comets orbiting or ejected from other stars. Further, if collisions between comets and NSs produce GRBs, then most NS - comet collisions in the Galaxy should arise from collisions between field NSs and interstellar comets. These Extra-Solar Oort Cloud (ESOC) burst sources should produce space-correlated repeaters whose events exhibit a wide range of peak luminosities and luminosity time histories, suggestive of the soft gammaray repeaters (SGRs). Since the Galactic ESOC population is expected to reflect the geometry of the intermediate-population Galactic "thick disk" (Gilmore, Wyse, \& Kuijken 1989), one expects a Galactic distribution for SGRs if comet - NS collisions are the burst mechanism. These general characteristics, and our rough estimate of 0.65 events $\mathrm{yr}^{-1}$ per source predicted for the NS - OC scenario, are qualitatively similar to the those of SGRs (Kouveliotou et al. 1993).

Based on the expected event rates, we are able to constrain the fraction of stars with Oort Clouds like our own to be no more than 4\%, unless either NS-comet collisions do not predominately produce $\gamma$-rays or the $\gamma$-rays are are highly beamed. If none of the detected SGRs known today lies within the BATSE-detectable distance scale for comet-NS events, the constraint on the fraction of stars with Oort Clouds like our own drops below $1 \%$.

ACKNOWLEDGEMENTS. We thank Hal Levison, Martin Duncan, Piero Madau, Serge Pineault, John Wang, and Paul Weissman for useful comments. We also thank an anonymous referee for making comments that strengthened our arguments for comet capture. 
This work was supported by the NASA Origins of Solar Systems program under NAGW3023 and by the NASA Astrophysical Theory Program at the University of Colorado (NAGW-766). 


\section{REFERENCES}

Atteia, J.-L., et al. 198T. ApJ. 320. L105

Bahcall. J. N. 1984. ApJ. 276. 169

Bhattacharya. D., Wijers, R. A. M. J., Hartmann, J. W.. \& Verbunt. F. 1992, A\&A. 254, 198

Blaes. O.. \& Madau. P. 1093. ApJ, 403. 690

Chanmugam, G. 1992, ARA\&A, 30, 143

Colgate, S. A., \& Petschek, A. G. 1981, ApJ, 248, 771

Gilmore, G., Wyse, R. F. G., \& Kuijken, K. 1989, ARA\&A, 27, 555

Gunn, J. E., \& Ostriker, J. P. 1970. ApJ, 160, 979

Harwit, M., \& Salpeter, E. E. 1973, ApJ, 186, L37

Helfand, D. J., \& Long, K. S. 1979, Nature, 282, 582

Higdon, J. C., \& Lingenfelter, R. 1990, ARA\&A, 28, 401

Hobbs, P. V. 1974, Ice Physics (Oxford: Oxford Univ. Press)

Hoyle, F., \& Lyttleton, R. A. 1941, MNRAS, 100, 27

Hughes, D. W. 1991, in Comets in the Post-Halley Era, Vol. 2, ed. R. L. Newburn et al. (Dordrecht: Kluwer), 825

Hurley, K. 1993, Nature, 365, 13

Katz, J. I. 1986, ApJ, 309, 253

Kouveliotou, C., et al. 1993, Nature, 362, 728

Kulkarni, S. R. 1986, ApJ, 306, L85

Kulkarni, S. R., \& Frail, D. A. 1993, Nature, 365, 33

Kulkarni, S. R., Frail, D. A., Kassim., N. E., Murakami, T., \& Vashist, G. 1994, Nature, 368,129

Livio, M., \& Taam, R. E. 1987, Nature, 327, 398 
Lyne, A. G.. Anderson, B.. \& Salter, M. J. 1982, MNRAS. 201. 503

Lyne. A. G.. Manchester. R. N.. \& Taylor. J. H. 1985, .MNR.AS. 213, 613

Lyne, A. G.. \& Lorimer, D. R. 1994, Nature. 369, 127

Mazets, E., Golenetski. S.. Guryan, Yu., \& Ilyinski. V. 1982, Ap. Space Sci.. \$4, 173

McDonnell, J. A. M., et al. 1993, Nature, 362, 732

McGlynn. T. A., \& Chapman, R. D., ApJ, 346, L105, 1989

Meegan, C. A., et al. 1992, Nature, 355, 143

Mitrofanov, I. G. 1990. Ap. Sp. Sci., 165, 137

Mitrofanov, I. G., \& Sagdeev, R. Z., 1990. Nature, 344. 313

Mumma, M. M.. Weissman. P. R., \& Stern, S. A. 1093. in Protostars \& Planets III, eds.

G. Levy, J. I. Lunine (Tucson: U. of Arizona Press), 1177

Narayan, R.. \& Ostriker, J. P. 1990, ApJ, 352, 222

Norris, J. P., et al. 1991, ApJ, 366, 240

Paczynski, B. 1991, Acta Astronomica, 41, 257

Paczynski, B. 1993, unpublished review

Pineault, S. 1990, Nature, 345, 233

Pineault, S. 1992, in Gamma-Ray Bursts: Observations, Analyses. and Theories, ed. C. Ho, R. I. Epstein. E. E. Fenimore (Cambridge Univ. Press), 81

Pineault, S., \& Poisson, E. 1989, ApJ, 347, 1141

Quashnock, J. M., \& Lamb, D. Q. 1993, MNRAS, 265, L45

Rothschild, R. E., Kulkarni, S. R., \& Lingenfelter, R. E. 1994, Nature, 368, 432

Rutledge, R. E., \& Lewin, W. H. G. 1993, MNRAS, 265, L51

Schaefer, B. E. 1993a, private communication

Schaefer, B. E. 1993b, Nature, 367, 411

Scotti, J. V., \& Melosh, H. J. 1993, Nature, 365, 733 
Sekanina. Z. 1976. Icarus, 27, 123

Sekanina. Z. 1982. in Comets. ed. L. Wilkening (Tucson: U. of Arizona Press), 251

Sekanina, Z. 1993, Science. 262. 387

Smith, I. A., \& Lamb, D. Q. 1993, ApJ, 410. L23

Stern, S. A., 1990, PASP, 102, 793

Syer, D., \& Saha, P. 1994, ApJ, 427, 714

Tremaine. S. 1993, in Planets Around Pulsars, ed. J. A. Phillips, S. E. Thorsett, S. R. Kulkarni (San Francisco: ASP Conference Series), 335

Tremaine, S., \& Żytkow, A. N. 1986, ApJ, 301, 155

Van Buren, D. 1981, ApJ, 249. 297

Wang, V. C., \& Lingenfelter, R. E. 1993, ApJ, 416, L13

Wang, J. \& Sutherland, R. S. 1994, private communication

Weaver, H. A. et al. 1994, Science, 263, 787

Weissman, P. R. 1990, Nature, 344, 825

Wetherill, G. W. \& ReVelle, D. O. 1982, in Comets, ed. L. Wilkening (Tucson: Univ. of Arizona Press), 297 